REVIEW SERIES

\title{
Challenges in pulmonary fibrosis - 1: Use of high resolution CT scanning of the lung for the evaluation of patients with idiopathic interstitial pneumonias
}

\author{
Michael B Gotway, Michelle M Freemer, Talmadge E King Jr
}

Thorax 2007;62:546-553. doi: 10.1136/thx.2004.040022

High resolution CT (HRCT) scanning has contributed significantly to the evaluation of patients with interstitial lung disease and is particularly useful in the diagnosis of idiopathic pulmonary fibrosis (IPF). The characteristic radiographic features of the idiopathic interstitial pneumonias on HRCT scans have been increasingly analysed and are now fairly well described. Based on current data, HRCT scanning can provide a confident, highly specific diagnosis of IPF in many patients with diffuse lung disease. This article reviews an organised approach to HRCT scanning and identifies the features that allow an accurate diagnosis of the idiopathic interstitial pneumonias to be made. The role of surgical lung biopsy is discussed in the diagnosis of cases when a definite HRCT diagnosis cannot be made.

See end of article for authors' affiliations

Correspondence to: Dr Michael Gotway, Scottsdale Medical Imaging, and affiliate of Southwest Diagnostic Imaging, 3501 North Scottsdale Road, Suite 130, Box 1573, Scottsdale, AZ 85251, USA

Received31 December 2004 Accepted 21 April 2005
$\mathrm{T}$ he diagnostic evaluation of a patient with dyspnoea presents a challenge to the clinician. Histopathological evaluation has shown that the clinical and radiological diagnosis of the idiopathic interstitial pneumonias (IIPs) is more heterogeneous than previously thought. The subclassification of the IIPs based on clinical and pathological criteria has important therapeutic and prognostic implications.

A structured, clinical, radiographic and pathological approach to the diagnosis of the IIPs is important in ensuring proper diagnosis and management of these patients. The most important distinction is the presence of usual interstitial seen in idiopathic pulmonary fibrosis (IPF). As surgical lung biopsy is a morbid procedure, there has been significant interest in exploring the clinical diagnosis of IPF. Historically the clinical diagnosis has been quite non-specific, but recent advances in the clinical and radiographic description of IPF have dramatically improved the clinicoradiographic diagnostic accuracy. The focus of this review is to clarify the role of high resolution computed tomography (HRCT) and surgical lung biopsy in the assessment of patients with IIP.

\section{ROLE OF HIGH RESOLUTION CT (HRCT) SCANNING IN THE DIAGNOSIS OF IDIOPATHIC INTERSTITIAL PNEUMONIAS (IIPS)}

The IIPs are comprised of UIP, non-specific interstitial pneumonia (NSIP), acute interstitial pneumonia (AIP), respiratory bronchiolitis-interstitial pneumonia (UIP), the histopathological pattern lung disease (RB-ILD), desquamative interstitial pneumonia (DIP), cryptogenic organising pneumonia (COP) and lymphocytic interstitial pneumonia (LIP). When evaluating a patient with dyspnoea for the possibility of an IIP, the history remains critical to eliminate any systemic diseases or potential drug or environmental exposures that may cause pulmonary disease. The physical examination can also serve to evaluate the possibility of a systemic illness. ${ }^{1}$

The conventional chest radiograph is of limited value in the diagnosis of IPF. Given its high sensitivity as a diagnostic test, HRCT scanning of the lung has become an indispensable tool to identify the presence of interstitial lung disease (ILD). Furthermore, in a patient who has an idiopathic ILD, as determined by the history and physical examination, HRCT evaluation also has the potential to narrow the differential diagnosis or to make a specific diagnosis. An organised approach to HRCT scanning techniques and interpretation in these patients is required for proper diagnosis and management.

\section{HRCT TECHNIQUE \\ Collimation}

Narrow collimation (section thickness) is required, usually of the order of $1 \mathrm{~mm}$, to achieve maximal spatial resolution. HRCT imaging is performed using axial, as opposed to helical, technique. A typical HRCT protocol uses 1-2 mm collimation every $10-20 \mathrm{~mm}$ throughout the thorax, which effectively images only approximately $10 \%$ of the lung parenchyma. Because HRCT scanning is typically used for the assessment of diffuse lung disease, such a sampling technique provides adequate representation of the disease process while minimising the radiation dose delivered to the patient. Some investigators have recently applied narrow collimation multi-slice helical CT scanning to patients with diffuse lung disease, but the benefit of volumetric imaging must be weighed against the substantial increased radiation dose inherent to this technique.

Abbreviations: AIP, acute interstitial pneumonia; COP, cryptogenic organising pneumonia; DIP, desquamative interstitial pneumonia; HRCT, high resolution CT; IIP, idiopathic interstitial pneumonia; ILD, interstitial lung disease; IPF, idiopathic pulmonary fibrosis; LIP, lymphocytic interstitial pneumonia; NSIP, non-specific interstitial pneumonia; RB-ILD, respiratory bronchiolitis-interstitial lung disease; UIP, usual interstitial pneumonia 

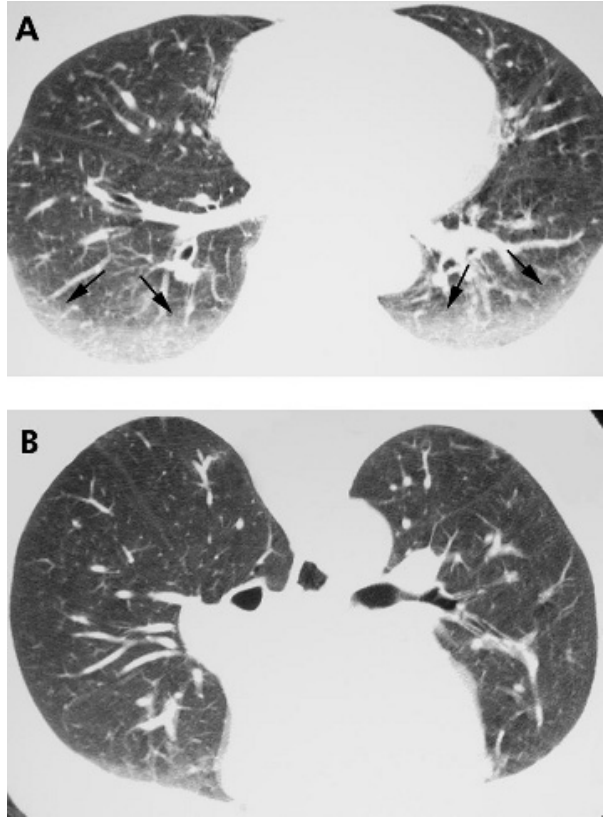

Figure 1 Value of prone high resolution (HR) CT imaging: resolution of dependent lung opacity. (A) Axial supine HRCT image showing bilateral dependent ground glass opacity (arrows; compare with fig 2A). (B) Axial prone HRCT image shows resolution of the ground glass opacity in (A), consistent with atelectasis. Note the opacity within the lungs anteriorly, representing dependent atelectasis occurring in the prone position.

\section{Expiratory scanning}

Integral to all HRCT scanning protocols is the use of expiratory scanning. Expiratory HRCT may be performed using static methods (imaging at functional residual capacity), lateral decubitus $\mathrm{CT}^{2}{ }^{2}$ or dynamic expiratory HRCT (imaging during a forced vital capacity manoeuvre). ${ }^{3}$ While expiratory HRCT often adds little to the evaluation of patients with IIPs (with the exception of RB-ILD), ${ }^{4}$ expiratory HRCT is very important for the evaluation of a number of other diffuse lung diseases that may clinically resemble IIPs, such as hypersensitivity pneumonitis.

\section{Prone imaging}

For many patients with ILD, and in particular for the IIPs, prone imaging is essential. Supine HRCT images commonly show opacities in the dependent portions of the lung (fig l) which are often caused by atelectasis. Such opacities may, however, resemble infiltrative lung disease (fig 2A). When patients are scanned in the prone position, atelectasis will resolve (fig 1B), unlike true lung pathology which will not resolve (fig $2 \mathrm{~B}$ ).

\section{APPROACH TO HRCT EVALUATION IN PATIENTS WITH IIPS}

HRCT findings of IIPs may be broadly categorised into foci of increased opacity and foci of decreased opacity (table 1). The major imaging findings manifesting as increased opacity on HRCT imaging in patients with IIPs include ground glass opacity, consolidation, linear and reticular opacities, architectural distortion, airway thickening and, occasionally, centrilobular nodules. While each of these findings individually may be seen in the various IIPs, certain findings tend to predominate more than others in each disease, as will be discussed subsequently. The imaging findings in patients with IIP that manifest as decreased opacity on HRCT imaging include traction bronchiectasis, honeycomb lung and air trapping (also,

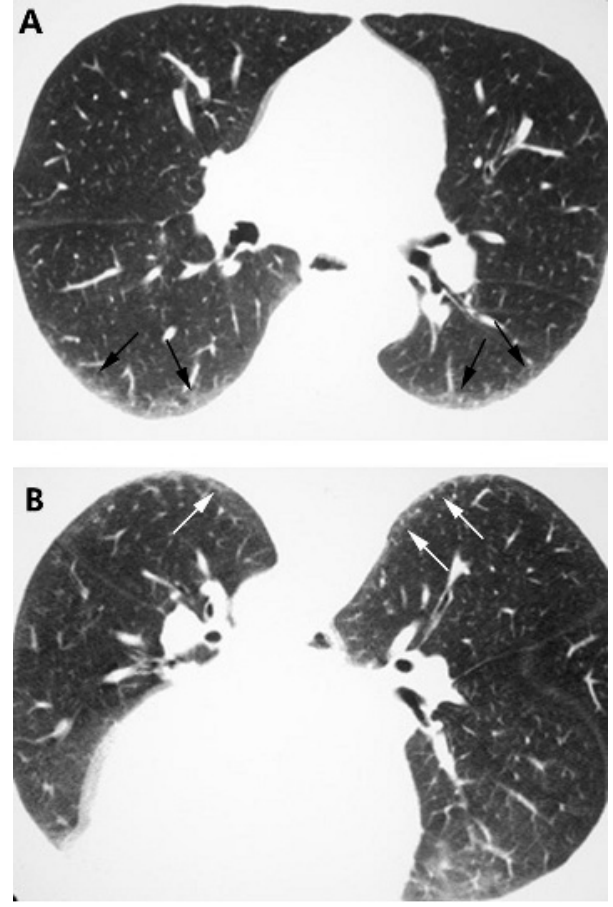

Figure 2 Value of prone high resolution (HR) CT imaging: persistence of infiltrative lung disease. (A) Axial supine HRCT image shows bilateral dependent ground glass opacity and reticulation (arrows). Note similarity to fig 1A. (B) Axial prone HRCT image showing persistence of the ground glass opacity and reticulation (arrows) seen in (A), consistent with infiltrative lung disease. Surgical lung biopsy proved non-specific interstitial pneumonitis.

Table 1 HRCT findings commonly encountered in patients with idiopathic interstitial pneumonias (IIPs)*

\begin{tabular}{|c|c|c|}
\hline IIP & Distribution & Predominant HRCT findings \\
\hline UIP & Basilar, subpleural & $\begin{array}{l}\text { Reticulation (usually coarse) } \\
\text { Architectural distortion } \\
\text { Traction bronchiectasis } \\
\text { Honeycombing }\end{array}$ \\
\hline NSIP & Basilar, subpleural & $\begin{array}{l}\text { Ground glass opacity } \\
\text { Reticulation (usually fine) } \\
\text { Architectural distortion } \\
\text { Traction bronchiectasis } \\
\text { Consolidation }\end{array}$ \\
\hline AIP & Diffuse & $\begin{array}{l}\text { Ground glass opacity } \\
\text { Consolidation }\end{array}$ \\
\hline RB-ILD & Multifocal & $\begin{array}{l}\text { Ground glass opacity } \\
\text { Airway thickening } \\
\text { Air trapping } \\
\text { Ground glass centrilobular nodules }\end{array}$ \\
\hline DIP & Multifocal, often peripheral & $\begin{array}{l}\text { Ground glass opacity } \\
\text { Reticulation }\end{array}$ \\
\hline $\mathrm{COP}$ & Subpleural, peribronchiolar & $\begin{array}{l}\text { Consolidation } \\
\text { Ground glass opacity }\end{array}$ \\
\hline LIP & No predominance & $\begin{array}{l}\text { Ground glass opacity } \\
\text { Peribronchiolar nodules } \\
\text { Centrilobular nodules } \\
\text { Linear opacities } \\
\text { Cysts }\end{array}$ \\
\hline
\end{tabular}

$\mathrm{HRCT}$, high resolution $\mathrm{CT}$; IIP, idiopathic interstitial pneumonia; UIP, usual interstitial pneumonia; NSIP, non-specific interstitial pneumonia; AIP, acute interstitial pneumonia; RB-ILD, respiratory bronchiolitis-interstitial lung disease; DIP, desquamative interstitial pneumonia; COP, cryptogenic organising pneumonia; LIP, lymphocytic interstitial pneumonia

*The predominant findings may vary somewhat depending on the phase of the disease. 


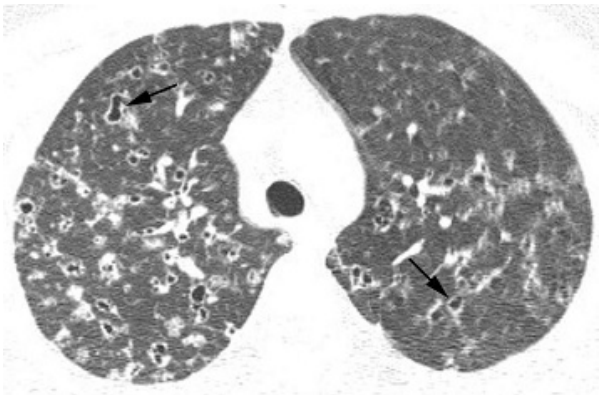

Figure 3 Patient with suspected idiopathic interstitial pneumonia: alternative diagnosis established with high resolution (HR) CT imaging. Axial HRCT image through the pulmonary apices shows bilateral bizarreshaped cysts (arrows) and nodules characteristic of Langerhans' cell histiocytosis.

because many of these patients are smokers, emphysema is a common finding).

The first step in the interpretation of HRCT imaging in patients with IIP is the assessment for the presence or absence of a pattern suggestive of UIP. ${ }^{1}$ If HRCT findings typical of UIP are found, one may confidently suggest the diagnosis of UIP and surgical lung biopsy may be avoided. In the absence of clear findings suggesting UIP, the HRCT scan should be examined for features that specifically indicate an alternative diagnosis, either another IIP or another interstitial lung process. The imaging differential diagnosis is then ordered accordingly, and the HRCT scan findings are correlated with the clinical history ${ }^{1}$ to suggest the most likely diagnosis. Occasionally a confident alternative diagnosis may be made in this situation (fig 3 ), but surgical lung biopsy is often required to firmly establish a diagnosis.

\section{USUAL INTERSTITIAL PNEUMONIA (UIP) Typical HRCT features}

HRCT findings characteristic of UIP consist of bilateral basilar subpleural reticulation often accompanied by traction bronchiectasis and architectural distortion and honeycomb cysts (fig 4). ${ }^{15-7}$ In general, these findings gradually decrease in extent from base to apex. The linear and reticular opacities are usually coarser in patients with UIP than those with other IIPs.

\section{Honeycombing in UIP}

One of the key findings (see below) that suggests the diagnosis of UIP on HRCT imaging is the presence of honeycomb cysts in a basilar subpleural distribution. Honeycomb changes appear on the HRCT scan as variably-sized cystic spaces that share walls and frequently stack upon one another in several layers (fig 4). The presence of centrilobular emphysema can sometimes make the diagnosis of honeycombing more difficult.

\section{Atypical HRCT features}

In addition to these typical findings, UIP is also characterised by the absence of a number of features. More specifically, ground glass opacity (particularly if extensive), consolidation, nodules, pleural effusion and lymphadenopathy are not conspicuous findings on the HRCT scan in patients with UIP. ${ }^{5}$ Mildly enlarged lymph nodes are often encountered on HRCT examinations performed on patients with UIP-IPF, but the presence of such lymph nodes does not contribute to making the diagnosis of UIP on imaging.

Importantly, the presence of ground glass opacity on the HRCT scan does not rule out histopathologically proven UIP but its presence-particularly if extensive-should suggest another diagnosis. The histological correlate of ground glass opacity on

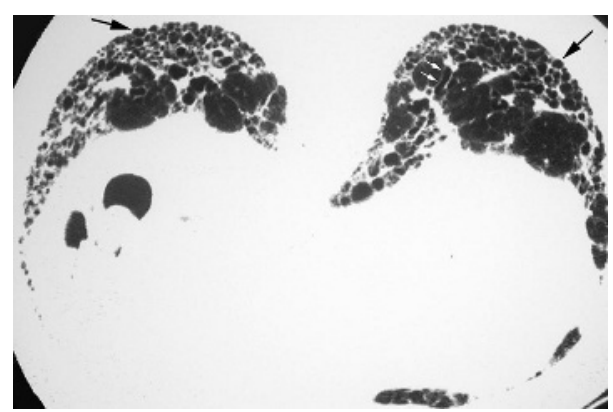

Figure 4 Usual interstitial pneumonia (UIP) pattern on high resolution (HR) CT imaging: characteristic findings. Axial prone HRCT image through the lung bases shows bilateral subpleural cysts (arrows) representing honeycombing, as well as reticulation and traction bronchiectasis (small white arrows).

HRCT imaging is often an inflammatory process within the interstitium or alveolar spaces. However, ground glass opacity is not synonymous with inflammation; rather, ground glass opacity on HRCT scans obtained in patients with UIP may reflect the presence of interstitial fibrosis on histological examination. ${ }^{89}$ In this setting, ground glass opacity will be accompanied by other findings suggestive of fibrosis such as architectural distortion, coarse reticulation, traction bronchiectasis and honeycombing.

\section{Accuracy of HRCT for the diagnosis of UIP ATS/ERS criteria}

Several studies have been performed to investigate whether HRCT scanning can obviate the need for surgical lung biopsy to make the diagnosis of UIP. The current ATS/ERS consensus statement regarding the diagnosis of IPF includes bibasilar reticular abnormalities with minimal ground glass opacities as one of the major criteria that can be used in the appropriate clinical context. The other criteria include no known causes of ILD, restrictive physiology and impaired gas exchange, and a transbronchial biopsy excluding alternative diagnoses in an individual over 50 years of age with the insidious onset of dyspnoea lasting longer than 3 months and crackles on examination.

\section{"Definite" UIP on HRCT}

In a group of patients from multiple centres selected on the basis of the suspicion of IPF, expert radiologists were able confidently to diagnose UIP in nearly $60 \%$ of patients with a

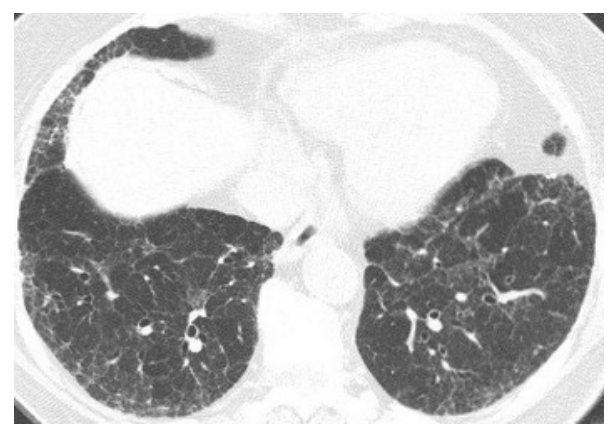

Figure 5 Pattern of usual interstitial pneumonia (UIP) on high resolution (HR) CT imaging: insufficiently characteristic findings. Axial HRCT image shows basilar subpleural reticulation but no evidence of honeycomb lung. A confident diagnosis of UIP could not be offered on the basis of the imaging findings. Surgical lung biopsy showed findings characteristic of UIP. 


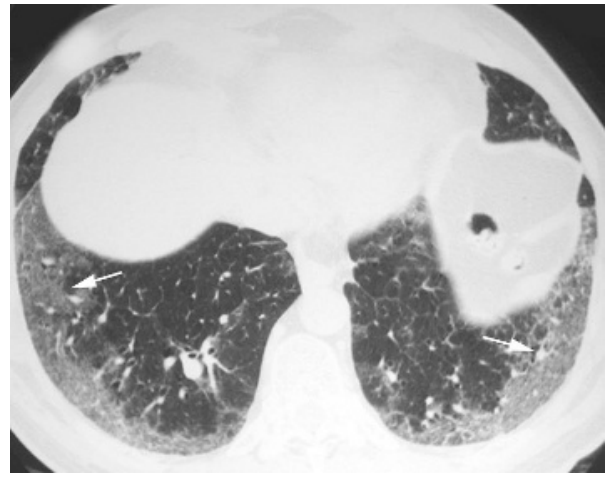

Figure 6 Pattern of non-specific interstitial pneumonia (NSIP) on high resolution (HR) CT imaging shows bilateral subpleural symmetric ground glass opacity (arrows).

positive predictive value of $96 \% .^{6}$ In a subsequent analysis, in the presence of the other typical findings of UIP described above, either of two particular findings-upper lobe reticulations and lower lobe honeycombing-was found to increase the probability of a histopathological diagnosis of UIP by 5-6-fold. ${ }^{5}$ Similarly, in another study of patients suspected of having an IIP, the presence of honeycombing on the HRCT scan in at least one lobe had a positive predictive value of $92 \% .{ }^{10}$ However, the diagnosis of UIP cannot be confidently offered on HRCT imaging when honeycomb cysts are not seen (fig 5). In fact, some patients without radiographic honeycombing will have UIP diagnosed on surgical lung biopsy. ${ }^{6}$

\section{Prognostic value of HRCT scanning in UIP}

In addition to its diagnostic value in UIP, HRCT scanning also has prognostic value. The presence of a definite (confident) HRCT diagnosis of UIP on the basis of basilar honeycombing portends a worse survival than for individuals without honeycombing on the HRCT scan and for those with a histopathological diagnosis of UIP. ${ }^{10}{ }^{11}$

\section{NON-SPECIFIC INTERSTITIAL PNEUMONIA (NSIP) Typical features}

The most difficult IIP to distinguish from a UIP is NSIP. However, in contrast to UIP, the dominant feature of NSIP on HRCT imaging is basilar, subpleural, symmetrical, bilateral ground glass opacity (fig 6). ${ }^{12-14}$ In fact, ground glass opacity may be the sole feature in nearly one-third of patients with NSIP. ${ }^{1}$ A peculiar pattern in which the peripheral ground glass opacity and reticulation spares the immediate subpleural region of lung has been recognised (fig 7) and is considered suggestive of NSIP. Irregular linear and reticular opacities are often present in patients with NSIP (fig 8), and these opacities become increasingly coarse as the fibrotic elements become more pronounced on histopathological specimens. ${ }^{14}$ Traction bronchiectasis and bronchiolectasis is often visible and also becomes increasingly prominent as the fibrosis progresses. ${ }^{14}$

\section{Honeycombing in NSIP}

Initial experience suggested that honeycomb changes are very uncommon in patients with NSIP, ${ }^{15}$ but larger studies have shown that honeycomb lung may be seen in as many as $27 \%$ of patients with NSIP. ${ }^{14}$ Patients with NSIP who have honeycomb cysts visible on the HRCT scan, however, usually have a more fibrotic (rather than a cellular) form of NSIP, and traction bronchiectasis, traction bronchiolectasis, coarse reticulation and architectural distortion are usually also evident. It should be noted that, given the frequent difficulty in distinguishing fibrotic NSIP from UIP pathologically, many of these cases with

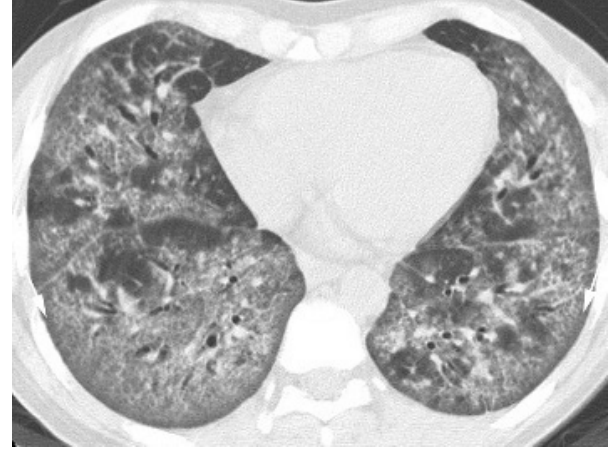

Figure 7 Pattern of non-specific interstitial pneumonia (NSIP) on high resolution (HR) CT imaging shows peripheral ground glass opacity sparing the immediate subpleural lung (arrows).

honeycomb lung are believed to represent cases of UIP-IPF (NSIP Workshop Committee. American Thoracic Society Workshop on Idiopathic Nonspecific Interstitial Pneumonia (NSIP), American Thoracic Society International Conference. Seattle, WA, 2003).

\section{Accuracy of HRCT scanning for the diagnosis of NSIP}

The reliability of a "confident" HRCT diagnosis of NSIP is more tenuous than for UIP. Even when cases are known to be either UIP or NSIP histopathologically, the diagnostic accuracy of radiologists when they are confident of the diagnosis of NSIP is only $72 \% .{ }^{16}$ In practice, such limited accuracy in a group of patients known to have a fibrotic IIP (chance alone would predict $50 \%$ accuracy) indicates the need for surgical lung biopsy to diagnose NSIP.

Serial HRCT images may show improvement in ground glass opacity and reticulation in patients with NSIP undergoing treatment (fig 9), although residual abnormalities often remain.

\section{ACUTE INTERSTITIAL PNEUMONIA (AIP)}

HRCT features can distinguish AIP from UIP. The typical HRCT features of AIP are bilateral, multifocal or diffuse areas of ground glass opacity and consolidation, usually without pleural effusion (fig 10). ${ }^{17}{ }^{18}$ No clear zonal distribution is identifiable, although the consolidation is often dependent in location. HRCT findings often reflect the stage of the disease and underlying histopathological process. During the organising phase of the disease, HRCT findings consistent with evolving fibrosis are often present including traction bronchiectasis, linear and reticular abnormalities, and architectural

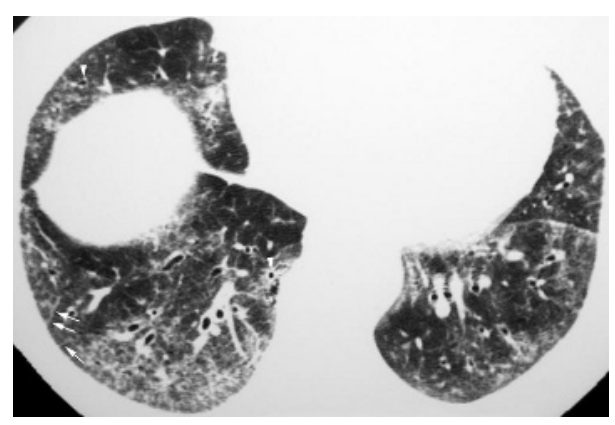

Figure 8 Pattern of non-specific interstitial pneumonia (NSIP) on high resolution (HR) CT imaging shows bilateral subpleural linear and reticular (arrows) opacities. Note the presence of traction bronchiolectasis (arrowheads). 


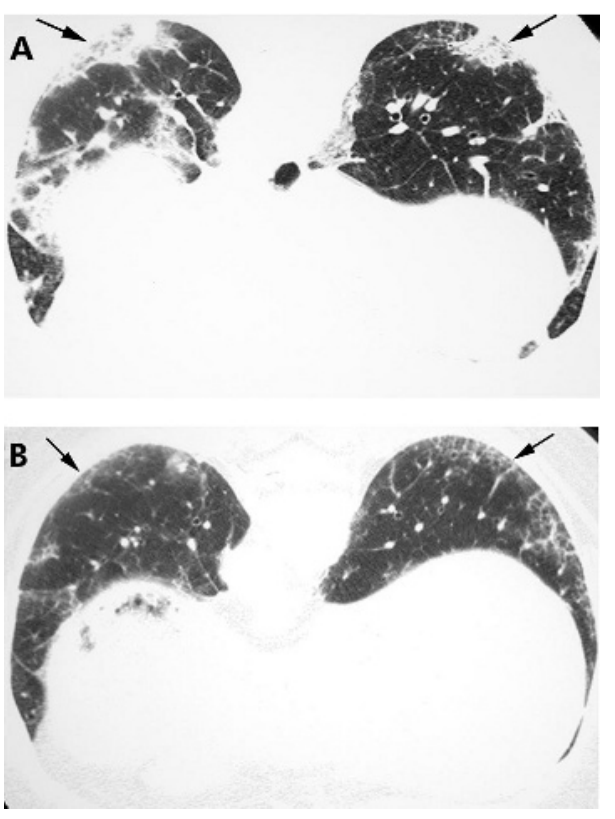

Figure 9 Non-specific interstitial pneumonia (NSIP) on high resolution (HR) CT imaging: improvement with treatment. (A) Axial HRCT image shows basilar subpleural ground glass opacity, reticulation and consolidation (arrows), proved to be NSIP on surgical biopsy. (B) Axial HRCT image obtained 3 months after immunosuppressive therapy showing regression of the ground glass opacity and consolidation; some linear and reticular elements (arrows) remain.

distortion. ${ }^{17}$ Among survivors, HRCT scans show clearing of most abnormalities, but foci of reticulation, parenchymal distortion, cystic change or honeycombing may remain. ${ }^{17}{ }^{18}$

\section{SMOKING-RELATED INTERSTITIAL LUNG DISEASES: RESPIRATORY BRONCHIOLITIS-INTERSTITIAL LUNG DISEASE (RB-ILD) AND DESQUAMATIVE INTERSTITIAL PNEUMONIA (DIP)}

While a history of smoking is almost always present in RB-ILD and DIP, patients with UIP are also frequently smokers. However, the HRCT features of these smoking related ILDs show a combination of findings distinct from UIP, such as, prominent ground glass opacity, subpleural reticulation, emphysema and cystic changes that are not classic for honeycomb lung.

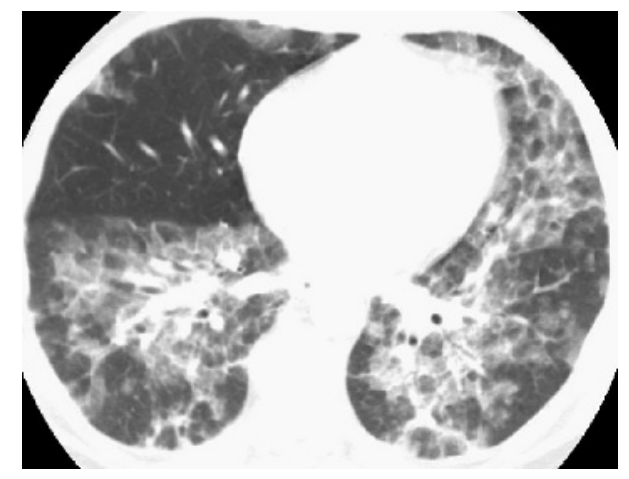

Figure 10 Acute interstitial pneumonia (AIP) on high resolution (HR) CT imaging. Axial HRCT image shows multifocal bilateral areas of ground glass opacity and bronchovascular thickening in a patient with AlP shown on surgical lung biopsy.

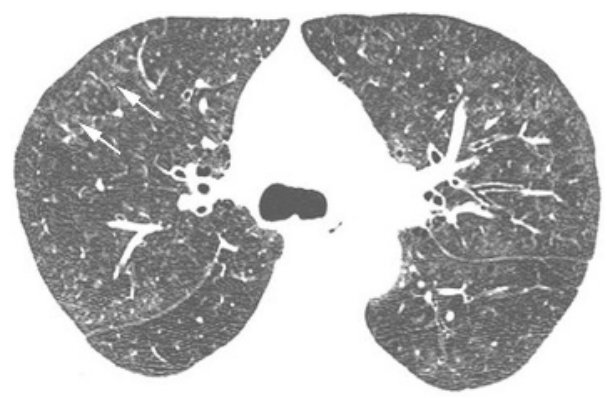

Figure 11 Respiratory bronchiolitis-interstitial lung disease (RB-ILD) on high resolution (HR) CT imaging: ground glass attenuation centrilobular nodules. Axial HRCT image shows multiple ground glass attenuation nodules (arrows) and patchy areas of ground glass opacity.

\section{Respiratory bronchiolitis-interstitial lung disease (RB- ILD)}

The primary HRCT findings in patients with RB-ILD include centrilobular ground glass attenuation nodules (fig 11), patchy areas of ground glass opacity (fig 12) and airway thickening. ${ }^{14} 1920$ Patchy areas of hypoattenuation (fig 12) representing mosaic perfusion are often encountered, and may be shown to represent air trapping on expiratory imaging. ${ }^{4}$ These findings may resolve with treatment. Centrilobular emphysema is often present in patients with RB-ILD.

\section{Desquamative interstitial pneumonia (DIP)}

Desquamative interstitial pneumonia (DIP) primarily manifests on HRCT imaging as multifocal or diffuse ground glass opacity, often with a basilar and peripheral predominance (fig 13). ${ }^{19}{ }^{21}$ Irregular linear and reticular opacities are commonly seen but are not usually the dominant features of the disease. Honeycombing may be seen in the minority of patients and is usually limited in extent.

\section{CRYPTOGENIC ORGANISING PNEUMONIA (COP) \\ Typical features}

COP is also usually radiographically distinct from UIP, with the most common HRCT finding being consolidation which is present in $90 \%$ of patients. ${ }^{22}$ In up to $50 \%$ of patients the consolidation is peripheral or peribronchiolar in distribution (fig 14) ${ }^{1}$ and is more commonly encountered in the lower lobes. Mild bronchial dilation may be present in the areas of consolidation. Ground glass opacity is also commonly present

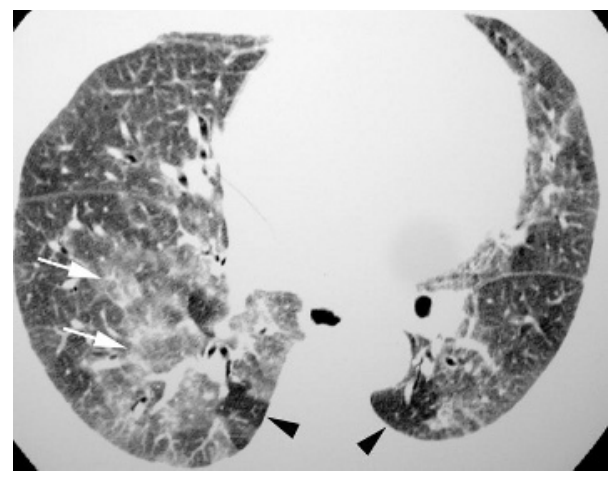

Figure 12 Respiratory bronchiolitis-interstitial lung disease (RB-ILD) on high resolution (HR) CT imaging: patchy ground glass opacity. Axial HRCT image shows multifocal areas of ground glass opacity (arrows). Hypoattenuating areas (arrowheads) have a noticeably lobular configuration and represent mosaic perfusion due to small airway obstruction. 


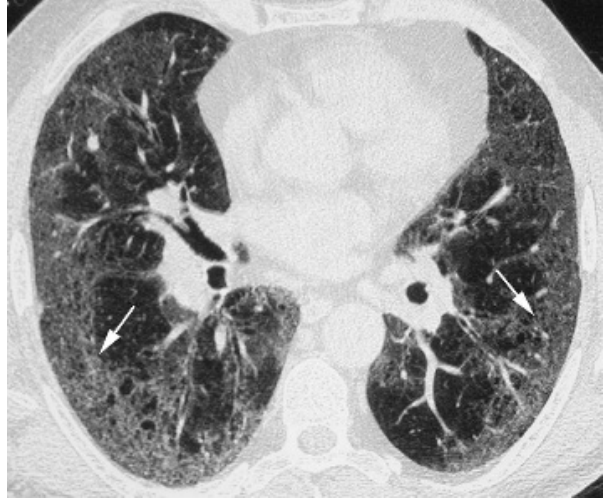

Figure 13 Desquamative interstitial pneumonia (DIP) on high resolution (HR) CT imaging. Axial HRCT image shows multifocal areas of ground glass opacity (arrows) in a peripheral distribution. Some reticulation is also evident.

and often coexists with consolidation. Small nodules are commonly seen in patients with COP, but are not usually a dominant feature.

\section{Atypical features of COP on the HRCT scan}

Several variant presentations of COP have been described. In $15 \%$ of patients COP may present as multiple masses, often with poorly circumscribed margins and an air bronchogram (fig 15). ${ }^{23}$ Patients with COP may also show the "reverse ground glass halo" sign, or "atoll" sign, on HRCT imaging. This finding consists of a nodular area of increased attenuation consisting of peripheral consolidation surrounding central ground glass attenuation (fig 16). While this finding is not specific for COP, nodules with this morphology are suggestive of COP in the appropriate clinical setting. Finally, a perilobular pattern of linear increased attenuation has been described..$^{25}$ This finding represents increased attenuation along the peripheral aspects of the secondary pulmonary lobule and superficially resembles interlobular septal thickening. The perilobular pattern was reported to be present in 57\% of patients with COP, often in association with other findings suggestive of COP. ${ }^{25}$ Although uncommon, linear and reticular abnormalities may be the dominant finding on HRCT imaging in patients with $\mathrm{COP}{ }^{26}$

COP presenting as parenchymal consolidation, ground glass opacity or nodules frequently regresses with treatment. ${ }^{122} 26$ However, COP presenting on the HRCT scan as linear

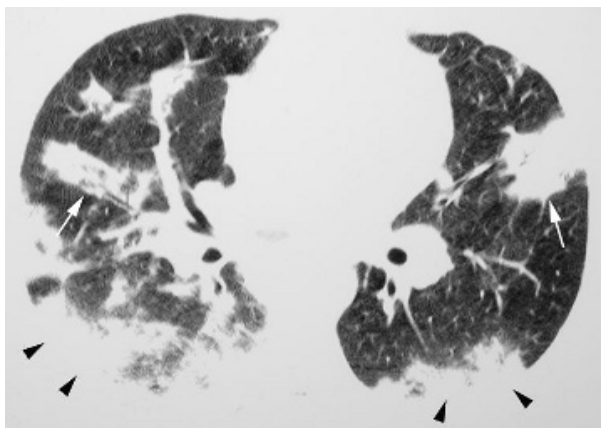

Figure 14 Cryptogenic organising pneumonia (COP) on high resolution (HR) CT imaging: peripheral and peribronchiolar distribution. Axial HRCT imaging shows consolidation in a peripheral (arrowheads) and peribronchiolar (arrows) distribution.
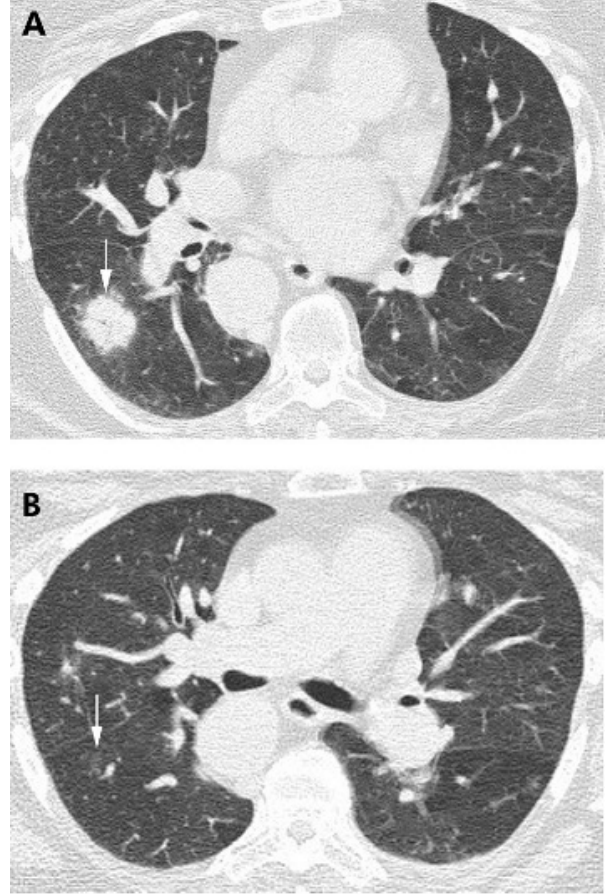

Figure 15 Cyrptogenic organising pneumonia (COP) on high resolution (HR) CT imaging: nodules with air bronchograms. (A) Axial HRCT imaging shows a poorly defined nodule with a central air bronchogram (arrow), shown on biopsy to represent COP. (B) Axial HRCT image performed several weeks after (A) showing near complete resolution of the nodule (arrow).

and reticular opacities frequently does not resolve following treatment. ${ }^{26}$

\section{LYMPHOCYTIC INTERSTITIAL PNEUMONIA (LIP) Typical features}

The HRCT findings of LIP are variable, but their lymphatic distribution along the peribronchovascular interstitium, interlobular septa and within the visceral pleura distinguishes this entity from UIP. Lymphoid interstitial infiltration along the centrilobular bronchus, within the centre of the secondary pulmonary nodule, will produce centrilobular nodules on the HRCT scan (fig 17). ${ }^{27}$ These nodules often show ground glass attenuation and are one of the more common manifestations of LIP on HRCT scanning. Occasionally these ground glass attenuation centrilobular nodules may become confluent and

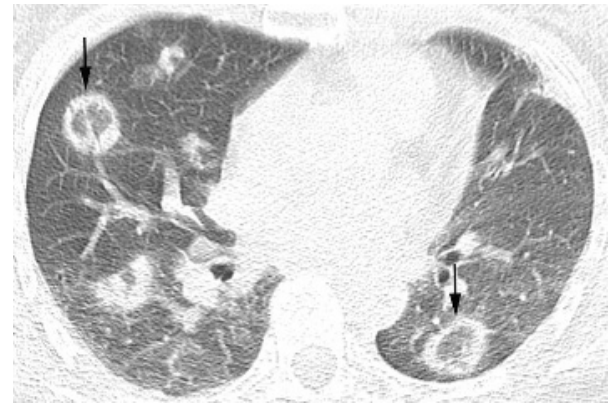

Figure 16 Cryptogenic organising pneumonia (COP) on high resolution (HR) CT imaging: the "reverse halo" or "atoll" sign. Axial HRCT imaging shows multiple nodular opacities consisting of consolidation peripherally and ground glass attenuation centrally (arrows). COP was proved at surgical lung biopsy. 


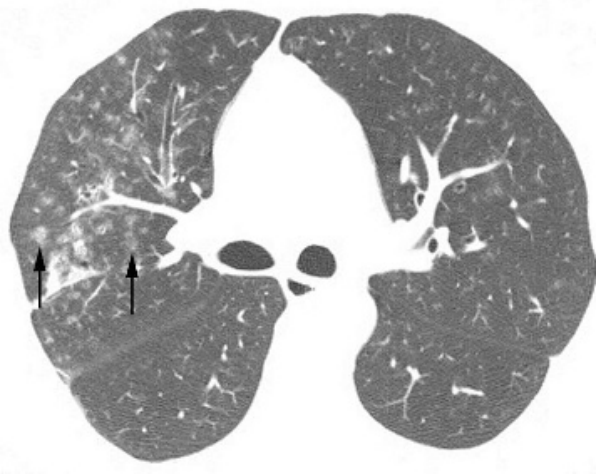

Figure 17 Lymphocytic interstitial pneumonia (LIP) on high resolution (HR) CT imaging: centrilobular ground glass attenuation nodules. Axial HRCT image shows poorly defined centrilobular ground glass attenuation nodules (arrows).

HRCT scans will then show multifocal areas of ground glass opacity in patients with LIP (fig 18). ${ }^{27}$ When lymphatic tissue along more central bronchovascular structures is primarily affected, HRCT scans will show more solid-appearing nodules studding vessels and bronchi (fig 19). Finally, involvement of the lymphatics within the visceral pleura will produce nodules along fissural and costal pleural surfaces (fig 20). ${ }^{27}$ Because interlobular septal infiltration is commonly found histopathologically in patients with LIP, interlobular septal thickening is often encountered on HRCT scans in patients with LIP. ${ }^{27}$

\section{Cysts in LIP}

Cystic spaces ranging in size from 1 to $30 \mathrm{~mm}$ have been reported in patients with LIP (fig 21).. ${ }^{28}$ These cysts are thought to be caused by bronchiolar obstruction and subsequent air trapping.

\section{SURGICAL LUNG BIOPSY}

Careful histopathological analysis allows the further classification of infiltrative lung diseases into distinct subgroups. In most settings, surgical lung biopsy has a diagnostic yield of $>90 \%$ and an overall morbidity and mortality of $\leqslant 2.5 \%$ and $\leqslant 0.3 \%$, respectively. ${ }^{29-31}$

Video-assisted thoracoscopic surgery lung biopsy has replaced thoracotomy in the diagnosis of ILD. There are three important situations in which thoracoscopic lung biopsy is contraindicated: inability to tolerate single lung ventilation (eg, severe hypoxaemia, high airway pressures), coagulopathy and pleural adhesions or scarring from previous thoracic surgery or pleurodesis. If a histological diagnosis is essential in these patients, a limited thoracotomy is usually the surgical approach of choice.

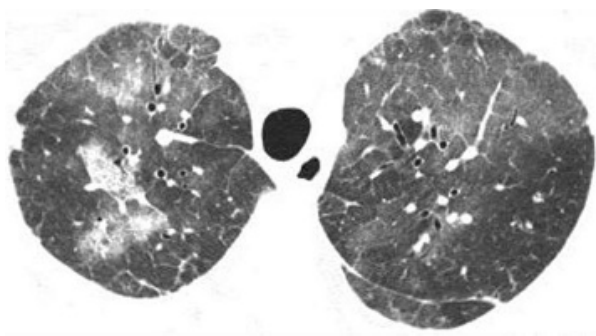

Figure 18 Lymphocytic interstitial pneumonia (LIP) on high resolution (HR) $\mathrm{CT}$ imaging: ground glass opacity. Axial HRCT scan shows multifocal areas of ground glass opacity shown to represent LIP on surgical lung biopsy.

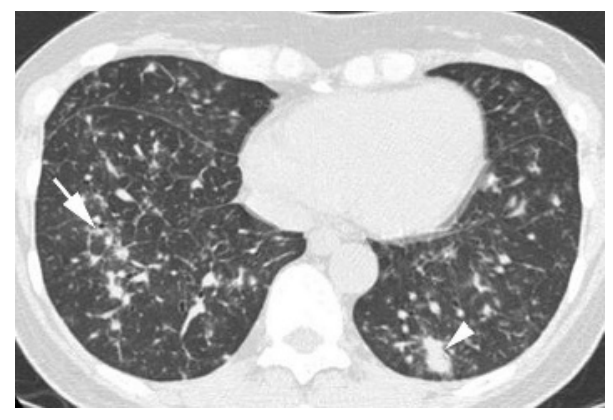

Figure 19 Lymphocytic interstitial pneumonia (LIP) on high resolution (HR) CT imaging: peribronchovascular nodules. Axial HRCT scan shows several nodules positioned along bronchi (arrow), consistent with a peribronchiolar location. Mild interlobular septal thickening and patchy areas of ground glass opacity are also seen in the right lower lobe. Peribronchiolar consolidation (arrowhead) is also present in the left lower lobe.

Surgical lung biopsy should only be pursued when a histopathological diagnosis will alter medical treatment and/ or provide important prognostic information. Patients who are too ill to tolerate or to benefit from potential treatments or patients who do not wish to receive potential treatments should generally not have surgical lung biopsies. Absolute cutoffs based on age or comorbidity should be avoided; the decision to forego surgical lung biopsy should be made on a case-by-case basis. ${ }^{32}$

\section{CONCLUSIONS}

IIP remains a source of confusion and consternation for clinicians. However, HRCT scanning has become a valuable tool allowing identification of the presence, extent and severity of ILD. Clinically, the distinction between UIP and all other (non-UIP) IIPs is the key determinant of a patient's course. Fortunately, when properly performed and evaluated by experienced thoracic radiologists, HRCT features alone are sufficient to make the diagnosis of UIP/IPF in $50-60 \%$ of cases selected based on the clinical suspicion of IPF. In the absence of peripheral bibasilar honeycombing with or without upper lobe reticulation-the features that allow radiologists to make a confident diagnosis of UIP-surgical lung biopsy is warranted. In this diagnostic process, the importance of both the clinical evaluation and radiological interpretation are critical. In the absence of a clear clinical and radiological diagnosis, we

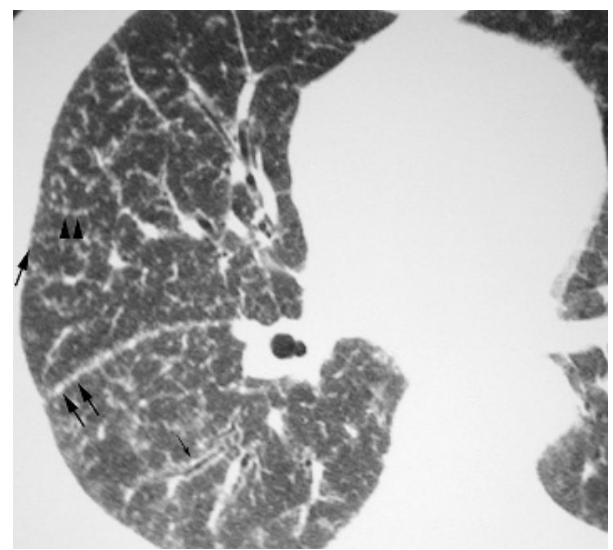

Figure 20 Lymphocytic interstitial pneumonia (LIP) on high resolution (HR) CT imaging: costal and fissural pleural nodules. Axial HRCT scan shows numerous well-defined nodules related to both costal and fissural pleural surfaces (arrows). 

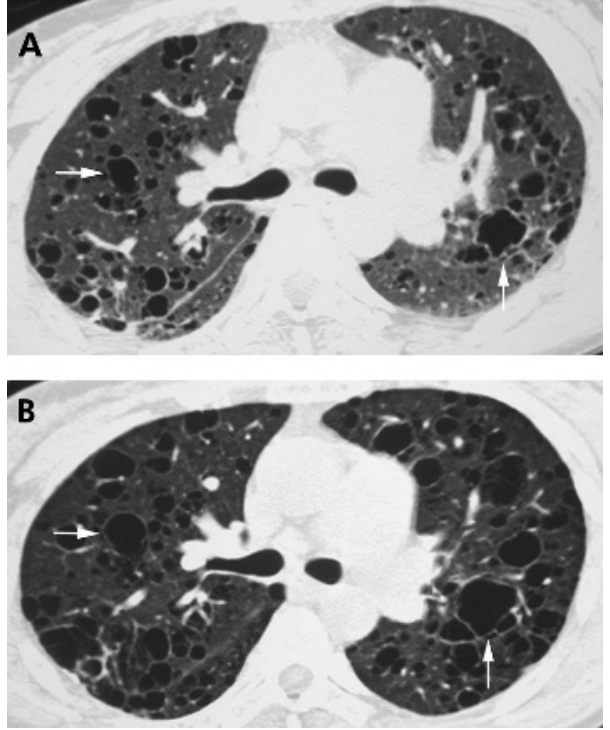

Figure 21 Lymphocytic interstitial pneumonia (LIP) on high resolution (HR) $C T$ imaging: cysts. (A) Axial HRCT scan showing several thin-walled cysts in a patient with LIP and Siögren's syndrome (arrows). (B) Axial HRCT scan obtained 2 years after (A) showing interval enlargement of these cysts (arrows).

recommend pursuing surgical lung biopsy when obtaining a histopathological diagnosis will alter medical treatment and/or provide important prognostic information. Knowledge of the underlying histopathology will allow for more accurate prognosis, help guide treatment, and make possible the clinical investigation of novel therapeutic agents for patients with IIP. ${ }^{32}$

\section{Authors' affiliations}

M B Gotway, Department of Radiology, University of California at San Francisco, and Division of Pulmonary and Critical Care Medicine, San Francisco General Hospital, San Francisco, California, USA

M M Freemer, T E King Jr, Department of Medicine, University of California at San Francisco, and Division of Pulmonary and Critical Care Medicine, San Francisco General Hospital, San Francisco, California, USA

Funding: None.

Competing interests: None.

\section{REFERENCES}

1 American Thoracic Society/European Respiratory Society International Multidisciplinary Consensus Classification of the Idiopathic Interstitial Pneumonias. Joint statement of the American Thoracic Society (ATS) and the European Respiratory Society (ERS) adopted by the ATS board of directors, June 2001 and by the ERS Executive Committee, June 2001. Am J Respir Crit Care Med 2002; 165:277-304.

2 Franquet T, Stern EJ, Gimenez A, et al. Lateral decubitus CT: a useful adjunct to standard inspiratory-expiratory CT for the detection of air-trapping. AJR Am J Roentgenol 2000;174:528-30.

3 Gotway MB, Lee ES, Reddy GP, et al. Low-dose, dynamic, expiratory thin-section CT of the lungs using a spiral CT scanner. J Thorac Imaging 2000;15:168-72.

4 Park JS, Brown KK, Tuder RM, et al. Respiratory bronchiolitis-associated interstitial lung disease: radiologic features with clinical and pathologic correlation. J Comput Assist Tomogr 2002;26:13-20.
5 Hunninghake GW, Lynch DA, Galvin JR, et al. Radiologic findings are strongly associated with a pathologic diagnosis of usual interstitial pneumonia. Chest 2003;124:1215-23.

6 Hunninghake GW, Zimmerman MB, Schwartz DA, et al. Utility of a lung biopsy for the diagnosis of idiopathic pulmonary fibrosis. Am J Respir Crit Care Med 2001;164:193-6.

7 Raghu G, Mageto YN, Lockhart D, et al. The accuracy of the clinical diagnosis of new-onset idiopathic pulmonary fibrosis and other interstitial lung disease: a prospective study. Chest 1999;116:1168-74.

8 Leung AN, Staples CA, Muller NL. Chronic diffuse infiltrative lung disease: comparison of diagnostic accuracy of high-resolution and conventional CT. AJR Am J Roentgenol 1991;157:693-6.

9 Lynch DA. Ground glass attenuation on CT in patients with idiopathic pulmonary fibrosis. Chest 1996;110:312-3.

10 Flaherty KR, Toews GB, Travis WD, et al. Clinical significance of histological classification of idiopathic interstitial pneumonia. Eur Respir J 2002;19:275-83.

11 Flaherty KR, Thwaite EL, Kazerooni EA, et al. Radiological versus histological diagnosis in UIP and NSIP: survival implications. Thorax 2003;58:143-8.

12 Park JS, Lee KS, Kim JS, et al. Nonspecific interstitial pneumonia with fibrosis: radiographic and CT findings in seven patients. Radiology 1995; 195:645-8.

13 Nagai S, Kitaichi $M$, Itoh $H$, et al. Idiopathic nonspecific interstitial pneumonia/ fibrosis: comparison with idiopathic pulmonary fibrosis and BOOP. Eur Respir J. 1998;12: 1010-9; erratum, 1999;13:711.

14 Johkoh T, Muller NL, Colby TV, et al. Nonspecific interstitial pneumonia: correlation between thin-section CT findings and pathologic subgroups in 55 patients. Radiology 2002;225:199-204.

15 Kim TS, Lee KS, Chung MP, et al. Nonspecific interstitial pneumonia with fibrosis: high-resolution CT and pathologic findings. AJR Am J Roentgenol 1998;171:1645-50.

16 MacDonald SL, Rubens MB, Hansell DM, et al. Nonspecific interstitial pneumonia and usual interstitial pneumonia: comparative appearances at and diagnostic accuracy of thin-section CT. Radiology 2001;221:600-5.

17 Ichikado K, Johkoh T, Ikezoe J, et al. Acute interstitial pneumonia: high-resolution CT findings correlated with pathology. AJR Am J Roentgenol 1997;168:333-8.

18 Johkoh T, Muller NL, Taniguchi H, et al. Acute interstitial pneumonia: thin-section CT findings in 36 patients. Radiology 1999;211:859-63.

19 Heyneman LE, Ward S, Lynch DA, et al. Respiratory bronchiolitis, respiratory bronchiolitis-associated interstitial lung disease, and desquamative interstitial pneumonia: different entities or part of the spectrum of the same disease process? AJR Am J Roentgenol 1999;173:1617-22.

20 Desai, SR, Ryan, SM, Colby TV. Smoking-related interstitial lung diseases: histopathological and imaging perspectives. Clin Radiol 2003;58:259-68.

21 Hartman TE, Primack SL, Swensen SJ, et al. Desquamative interstitial pneumonia: thin-section CT findings in 22 patients. Radiology 1993;187:787-90.

22 Lee KS, Kullnig P, Hartman TE, et al. Cryptogenic organizing pneumonia: CT findings in 43 patients. AJR Am J Roentgenol 1994; 162:543-6.

23 Akira M, Yamamoto S, Sakatani M. Bronchiolitis obliterans organizing pneumonia manifesting as multiple large nodules or masses. AJR Am J Roentgenol 1998;170:291-5

24 Bouchardy LM, Kuhlman JE, Ball WC, et al. CT findings in bronchiolitis obliterans organizing pneumonia (BOOP) with radiographic, clinical, and histologic correlation. J Comput Assist Tomogr 1993;17:352-7.

25 Ujita M, Renzoni EA, Veeraraghavan S, et al. Organizing pneumonia: perilobular pattern at thin-section CT. Radiology 2004;232:757-61.

26 Lee JS, Lynch DA, Sharma S, et al. Organizing pneumonia: prognostic implication of high-resolution computed tomography features. J Comput Assist Tomogr 2003;27:260-5.

27 Johkoh T, Muller NL, Pickford HA, et al. Lymphocytic interstitial pneumonia: thinsection CT findings in 22 patients. Radiology 1999;212:567-72.

28 Ichikawa Y, Kinoshita M, Koga T, et al. Lung cyst formation in lymphocytic interstitial pneumonia: CT features. J Comput Assist Tomogr 1994; 18:745-8.

29 Gaensler EA, Carrington CB. Open biopsy for chronic diffuse infiltrative lung disease: clinical, roentgenographic, and physiological correlations in 502 patients. Ann Thorac Surg 1980;30:411-26.

30 Bensard DD, Mclntyre RC Jr, Waring BJ, et al. Comparison of video thoracoscopic lung biopsy to open lung biopsy in the diagnosis of interstitial lung disease. Chest 1993;103:765-70.

31 Ayed AK, Raghunathan R. Thoracoscopy versus open lung biopsy in the diagnosis of interstitial lung disease: a randomised controlled trial. J R Coll Surg Edinb 2000:45:159-63.

32 Collard HR, King Jr TE. Clinical significance of histopathologic subgroups in the idiopathic interstitial pneumonias: is surgical lung biopsy essential? Semin Respir Crit Care Med 2001;22:347-56. 